\title{
A Small Perturbation CFD Method for Calculation of Seal Rotordynamic Coefficients
}

\author{
M. M. ATHAVALE and R. C. HENDRICKS \\ CFD Research Corporation, Huntsville, AL NASA Lewis Research Center, Cleveland, OH
}

\begin{abstract}
Seal rotordynamic coefficients link the fluid reaction forces to the rotor motion, and hence are needed in the stability calculations for the overall rotating systems. Presented in this paper is a numerical method for calculations of rotordynamic coefficients of turbomachinery seals with rotors nominally at centered, eccentric and/or misaligned position. The rotor of the seal is assumed to undergo a prescribed small whirling motion about its nominal position. The resulting flow variable perturbations are expressed as Fourier functions in time. The N-S equations are used to generate the governing equations for the perturbation variables. Use of complex variables for the perturbations renders the problem quasi-steady. The fluid reaction forces are integrated on the rotor surface to obtain the fluid reaction forces at several different whirl frequencies. The rotordynamic coefficients are calculated using appropriate curve fitting. Details of the model are presented, and sample results for concentric and eccentric annular incompressible flow seals are included to demonstrate the capability and accuracy of the proposed method.
\end{abstract}

Key Words: Seals; rotordynamics; turbomachinery; perturbation; Navier-Stokes; CFD

\section{INTRODUCTION}

\section{Seal Rotordynamics}

$\mathrm{T}$ urbomachinery seals interface between rotating parts such as rotors, blade tips, and stationary parts such as housings. The seals, used to isolate regions of different pressures, are non-contacting, and allow a leakage flow across. The fluid flow that exists in seals generates reaction forces on the rotor. As a rotor moves away from its nominal operating position, the fluid flow in the seal is altered and reaction forces are generated on the rotor. Knowledge of the type and magnitude of these forces is important when calculating the stability characteristics of the overall rotating system. The reaction forces can be destabilizing e.g. in a labyrinth seal, Alford [1965], or with proper designs, the reaction forces can be used to stabilize the rotor and provide load bearing capabilities in a seal, Von Pragenau [1982, 1987].

As a seal rotor moves away from its nominal position, the reaction forces generated by the fluid flow can be linked to the rotor displacement, velocity and accelerations using a spring-damper-mass system in 2 directions. For the seal configuration shown in Figure 1, the reaction forces $F_{y}$ and $F_{z}$ can be linked to the rotor motion using the relation

$$
\begin{aligned}
& -\left[\begin{array}{c}
F_{y} \\
F_{z}
\end{array}\right]=\left[\begin{array}{cc}
K_{y y} & K_{y z} \\
-K_{z y} & K_{z z}
\end{array}\right]\left[\begin{array}{l}
y \\
z
\end{array}\right]+\left[\begin{array}{cc}
C_{y y} & C_{y z} \\
-C_{z y} & C_{z z}
\end{array}\right]\left[\begin{array}{l}
\dot{y} \\
\dot{z}
\end{array}\right] \\
& +\left[\begin{array}{cc}
M_{y y} & M_{y z} \\
-M_{z y} & M_{z z}
\end{array}\right]\left[\begin{array}{l}
\ddot{y} \\
\ddot{z}
\end{array}\right]
\end{aligned}
$$

where $y, \dot{y}, \ddot{y}$ and $z, \dot{z}, \ddot{z}$ are the displacements, velocities, and accelerations in the $\mathrm{Y}$ and $\mathrm{Z}$ directions. $K_{y y}$ and $K_{z z}$ are the direct and $K_{y z}$ and $K_{z y}$ the cross-coupled stiffness coefficients, $C_{y y}, C_{z z}$ and $C_{y z}, C_{z y}$ direct and crosscoupled damping coefficients and $M_{y y}, M_{z z}$ and $M_{y z}, M_{z y}$ the direct and cross-coupled inertia (added mass) coefficients. Linked in this fashion, the rotordynamic coefficients can directly be used in the rotor stability calculations. When the nominal position of the rotor is concentric, the coefficient matrices become simpler and assume a skew-symmetric form, given as

$$
\begin{aligned}
& -\left[\begin{array}{c}
F_{y} \\
F_{z}
\end{array}\right]=\left[\begin{array}{cc}
K & k \\
-k & K
\end{array}\right]\left[\begin{array}{l}
y \\
z
\end{array}\right]+\left[\begin{array}{cc}
C & c \\
-c & C
\end{array}\right]\left[\begin{array}{l}
\dot{y} \\
\dot{z}
\end{array}\right] \\
& +\left[\begin{array}{cc}
M & m \\
-m & M
\end{array}\right]\left[\begin{array}{l}
\ddot{y} \\
\ddot{z}
\end{array}\right]
\end{aligned}
$$

with a total of only six distinct coefficients as against twelve for the general case. 


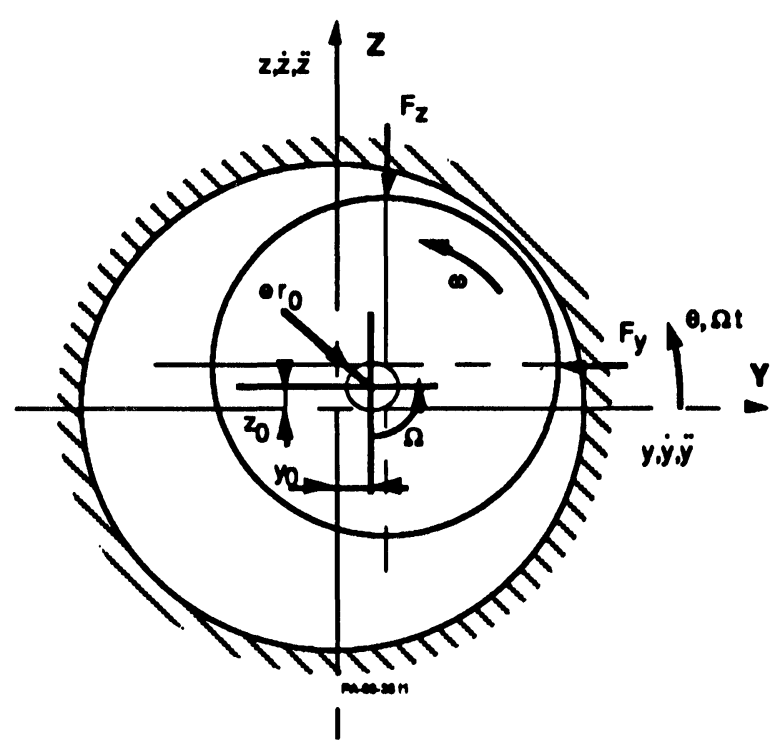

FIGURE 1 Geometry and nomenclature for a whirling rotor with small orbit radius

\section{Rotordynamic Coefficient Calculation Methods}

The influence of annular seal forces on the dynamics of centrifugal pumps was first investigated by Black [1969] and Black and Jensen [1970]. A bulk flow model was developed to compute the rotordynamic coefficients of annular seals. The theory treats the seal as a single lumped flow domain with no variations across the volume with friction factor correlations used to calculate wall shear. A perturbation in the rotor location is then used to generate the fluid reaction forces and subsequently rotordynamic coefficients. This method can be used to calculate only the skew-symmetric coefficient set. Bulk flow models based on Hirs' lubrication equation [1973] were developed by Childs for short [1983] and finite length [1983] annular seals. The bulk flow model was also extended to compressible flow seals by Nelson [1985] for annular and tapered seals.

Recently, more detailed solution procedures, based on 2-D solution procedures have been reported. The models account for flow variations in the circumferential as well as the axial direction but the variations across the film are integrated to give mean flow parameters. San Andres [1991] used the Hirs' lubrication equations with a 2-D solution procedure similar to the SIMPLE method for flow solutions in eccentric and misaligned seals and bearings under constant and variable properties. Simon and Frene [1989, 1991] used N-S equations integrated across the film thickness to treat eccentric annular seals.
In both the methods, the flow equations are perturbed to yield equations for the static flow and the perturbations in the flow. With a prescribed, small motion of the rotor, the perturbation variables are calculated and the pressures integrated on the rotor surface to generate fluid reaction forces and the rotordynamic coefficients.

The above described methods use integrated, mean values of the flow variables, averaged over either the overall flow domain, or across the fluid film in the seal. These models, then, can be expected to be adequate when the film thicknesses are small, and the flow is well behaved. When the seal has flow regions where there are steps, and/or regions with large film thicknesses e.g grooved or labyrinth seals, and/or when strong secondary motion is present e.g. in labyrinth seals, or as shown by Tam et. al. [1988], in eccentric annular seals, the average property models can be expected to deteriorate in accuracy. For such cases a full, 2- or 3-D, detailed analysis the flow becomes necessary for accurate flow and rotordynamic predictions.

With the advent of powerful computers, and sophisticated numerical techniques, the computational fluid dynamics (CFD) analyses of seal flows have become economical. Such a study was conducted by Tam et. al. [1988]. A 3-D Navier-Stokes analysis was done on an eccentric, whirling annular seal with incompressible flow. The fluid reaction forces were used to compute the rotordynamic characteristics of the seal. In addition, flow fields at several seal configurations and boundary conditions were computed. Computed flow fields showed the existence of recirculation bubbles in the seal flows under certain flow conditions. Nordmann et. al. [1987, 1989] have reported a finite-difference method based on the $\mathrm{N}-\mathrm{S}$ equations for seal rotordynamics, where they have used a small-perturbation analysis on the $\mathrm{N}-\mathrm{S}$ equations in cylindrical frame. The method was used to compute rotordynamics of nominally concentric, annular and grooved seals with incompressible and compressible flows. They also reported a study for eccentric, incompressible flow seals, Nordmann and Dietzen [1988]. More recently, Baskharone and Hensel [1991] have reported a 3-D finite element solution method, coupled with a perturbation analysis to compute flows and rotordynamics of nominally centered seals. They have also used this method to calculate the fluid reaction forces on rotating parts such as impellers.

Solution procedures based of the full CFD solutions for seal flow and rotordynamics have been reported by Athavale et. al. [1992]. One of the methods is based on a whirling rotor with a circular whirl orbit. A grid transformation is done to render the problem quasisteady, and solutions at several whirl frequencies are 
calculated. The rotor surface pressures are integrated to yield fluid reaction forces and appropriate curves are fit to the force-whirl frequency plot to obtain the rotordynamic coefficients. This method is similar to that used by Tam et. al. [1988]. This method is fast, but is applicable only for calculation of the skew-symmetric coefficients of a nominally concentric seal.

For nominally eccentric and/or misaligned seals, the full CFD solution method is based on the "shaker" method used in experimental determination of the rotordynamic coefficients (e.g. Childs et al [1996]). The rotor is moved along a radial direction from a nominally concentric or eccentric position, and the motion is sinusoidal in time. The resulting time-dependent flow is calculated using a moving grid algorithm. The pressures on the rotor surface are then integrated to provide time-accurate fluid reaction forces, which then are used to calculate the rotordynamic coefficients. This method, based on the full CFD solutions, is flexible and can be used for all nominal rotor positions, however, the computational times involved can be expected to be higher than the whirling rotor method, due to the time-accurate solutions that have to be obtained.

The method presented in this paper is developed to treat seals with rotors that are nominally eccentric and/or misaligned. To avoid the high computational times associated with the full, time accurate CFD solutions, the method is based on a small-perturbation analysis of the 3-D flow field in the eccentric seals. The methodology is similar to that described by Nordmann and Dietzen [1988], but the present approach uses the generalized Body Fitted Coordinate (BFC) formulation of the flow equations. For this reason, the present formulation is expected to be applicable to cylindrical seal problems as well as a variety of other problems with complicated shapes, e.g. blade tip flows and impeller flows.

\section{DESCRIPTION OF THE SOLUTION METHODOLOGY}

\section{Governing Equations}

The seal flow is described using the 3-D Navier-Stokes $(\mathrm{N}-\mathrm{S})$ equations in the generalized body-fitted-coordinate (BFC) system. The perturbations in the flow are introduced by assuming that the rotor center undergoes a whirl about its nominal, steady-state position. The rotor spin and whirl velocities are assumed to be $\omega$ and $\Omega$ respectively (see Figure 1). The rotor center position, in general, can be described as

$$
y=y_{0}+e r_{0} \cos \Omega t z=z_{0}+e r_{0} \sin \Omega t
$$

where $y_{0}$ and $z_{0}$ correspond to the nominal center location, $e$ is a small number, and $r_{O}$ is a suitable length scale, e.g. nominal seal clearance, $C_{0}$. The resultant time-varying flow variables are now expressed as, e.g.

$$
\begin{aligned}
& u=u_{0}+e u_{1} v=v_{0}+e v_{1} \\
& w=w_{0}+e w_{1} p=p_{0}+e p_{1}
\end{aligned}
$$

where the subscript 0 corresponds to the time-independent values that describe the steady flow in the seal with the rotor at its nominal position, and the time-dependent perturbations are denoted by the subscript 1 . Since the rotor center motion has sine and cosine functions of time, we assume that the time-dependent part of the resultant perturbations can also be expressed using Fourier series in time. Thus, we have, e.g.,

$$
\begin{aligned}
& u_{1}=u_{1 c} \cos \Omega t+u_{1 s} \sin \Omega t \\
& v_{1}=v_{1 c} \cos \Omega t+v_{1 s} \sin \Omega t
\end{aligned}
$$

where the values $u_{1 c}, u_{1 s}$ etc. now are functions of space only. When solutions of turbulent flows in the seals are considered, the variables connected with turbulence: turbulent kinetic energy $(k)$, dissipation rate $(\epsilon)$, and the turbulent viscosity $\left(\mu_{t}\right)$ are assumed to be unaffected by the rotor center motion, and hence are held constant at their steady-state, 0 th order values.

To derive the equations for perturbation variables, we start with the N-S equations, and introduce a coordinate transformation to map the time-varying grid locations $(\mathrm{x}, \mathrm{y}, \mathrm{z}, \mathrm{t})$ to a time-independent grid frame $(\xi, \eta, \zeta, \tau)$. The resulting equations are:

Continuity:

$$
\frac{\partial}{\partial \tau}(J \rho)+\frac{\partial}{\partial \xi^{k}}\left(J \rho \vec{V}_{g} \cdot \epsilon^{k}\right)+\frac{\partial}{\partial \xi^{k}}\left(J \rho \vec{V} \cdot \epsilon^{k}\right)=0
$$

Momentum:

$$
\begin{aligned}
& \frac{\partial}{\partial \tau}(J \rho \phi)+\frac{\partial}{\partial \xi^{k}}\left(J \rho \phi \vec{V}_{g} \cdot \epsilon^{k}\right)+\frac{\partial}{\partial \xi^{k}}\left(J \rho \phi \vec{V} \cdot \epsilon^{k}\right) \\
& =\frac{\partial}{\partial \xi^{k}}\left(\Gamma J g^{j k} \frac{\partial \phi}{\partial \xi^{\xi}}\right)+S_{\phi}
\end{aligned}
$$

where $\phi$ can be any of the Cartesian velocities $\mathrm{u}, \mathrm{v}$, or $\mathrm{w}$, $\xi^{\mathrm{k}}$ are the coordinate directions, $J$ is the Jacobian of transformation, $\epsilon^{\mathrm{k}}$ are the contravariant base vectors, $g^{j k}$ 
the elements of metric tensor and $\Gamma$ is the diffusion coefficient. $V_{g}$ is the grid velocity due to the rotor motion and is of order e. The term $\mathrm{S}_{\phi}$ contains pressure gradient terms and additional body force terms.

The definition of the dependent variables, Eqns. 4, are now used in the above equations. All terms containing powers of e higher than 1 are ignored, and then the terms with and without the factor e are separated to yield two sets of equations. The 0 th order equations now have no time-dependent terms or terms with grid velocity, and describe the steady-state seal flow. The 1 st order equations still have time-dependent terms. To render them quasi-steady, the Fourier expansions of the 1st order variables, Eqns. 5, are used in the 1st order equations, and the terms containing sine and cosine functions of time are separated out to yield independent flow equations for each of the $u_{1 c}, u_{1 s}$ etc. Since these variables are functions of space only, a steady-state solution treatment can be used to solve the equations for these 1st order variables. To avoid computations of, e.g. $\mathrm{u}_{1 \mathrm{c}}$ and $\mathrm{u}_{1 \mathrm{~s}}$ independently, we combine them to yield new variables:

$$
\hat{u}_{1}=u_{1 c}+i u_{1 s} \hat{v}_{1}=v_{1 c}+i v_{1 s} \text { etc }
$$

where $i=-1$ and $\hat{u}_{1},{ }_{1}$ now are complex variables. The flow equations for the complex variables are obtained in a similar manner, i.e.

$$
\left(\text { Eqn for } \hat{u}_{1}\right)=\left(\text { Eqn. for } u_{1 c}\right)+i\left(\text { Eqn for } u_{1 s}\right) \text { etc. }
$$

The flow equations for the 0th order, as stated before, are the steady-state $\mathrm{N}-\mathrm{S}$ equations and are not repeated here. The equations for the 1 st order variables and for incompressible flows are:

Continuity:

$$
\frac{\partial}{\partial \xi^{k}}\left(J \rho_{0} \vec{V}_{g}\right)+\frac{\partial}{\partial \xi^{k}}\left(J \rho_{0} \vec{V}_{1} \cdot \epsilon^{k}\right)=0
$$

where $\vec{V}_{g}$ is the complex grid velocity and $\vec{V}_{1}$ is the complex velocity vector.

Momentum:

$$
\begin{aligned}
& i \Omega \rho_{0} \cdot \hat{\phi}_{1} J+\frac{\partial}{\partial \xi^{k}}\left(J \rho_{0} \phi_{0} \vec{V}_{g} \cdot \epsilon^{k}\right)+\frac{\partial}{\partial \xi^{k}}\left(J \rho_{0} \hat{\phi}_{1} \vec{V}_{0} \cdot \epsilon^{k}\right) \\
& +\frac{\partial}{\partial \xi^{k}}\left(J \rho_{0} \phi_{0} \vec{V}_{1} \cdot \epsilon^{k}\right)=\frac{\partial}{\partial \xi^{k}}\left(J \Gamma g^{j k} \frac{\partial \hat{\phi}_{1}}{\partial \xi^{j}}\right)+\hat{S}_{1}^{\phi}
\end{aligned}
$$

with $\hat{\phi}_{1}=\hat{u}_{1}, \hat{V}_{1}$, or $\hat{W}_{1}$.

where the source term $\hat{S}_{1}^{\phi}$ now contains the 1 st order pressure gradient source terms, e.g. $\epsilon^{k} \partial \hat{p}^{1} / \partial \xi^{k}$ as well as the source terms that arise due to the grid motion. The grid motion terms contain complex grid metric coefficients, and the 0th order flow variables and hence are independent of the 1 st order flow variables.

\section{Solution Methodology}

The 0 th and 1 st order equations have a very similar structure, and can be solved using similar methodologies. The starting point for the solutions of the 0th order was an advanced code, SCISEAL, which has a finite volume integration scheme, uses Cartesian velocities are velocity variables in a colocated arrangement, and a pressure based-solution algorithm. The flow equations are solved sequentially, and the velocity-pressure coupling is provided using a modified SIMPLEC method. The code has capabilities of high spatial (up to 3rd order) and temporal (2nd order) accuracy, and has a variety of turbulence models (including the standard k- $\epsilon$, low-Re k- $\epsilon$, multiple scale $k-\epsilon$ and the 2-layer model) and treatment for isotropic surface roughness.

The 1st order flow module was added to SCISEAL to provide a seamless solution procedure for rotordynamics, which involved the following steps:

1. Solution of the steady-state equation for nominal rotor position.

2. Solution of 1 st order equations at 5 different whirl frequencies.

3. A least-square curve fitting algorithm for rotordynamic coefficients.

The 1st order equations are solved in a manner very similar to the 0th order equations. A sequential procedure is used to solve the equations, and pressure-velocity coupling is provided by the modified SIMPLEC algorithm. The major differences between the 1 st and the 0th order equations are: a) The 1st order equations are constant coefficient equations, and hence linear in nature. This affords faster convergence of the 1st order set, and b) The 1st order variables are complex quantities and separate modules are required to treat the equations. The complex arithmetic also tends to increase the computational times.

\section{Boundary Conditions}

Boundary condition specifications for the 1st order equations is straightforward. A Neumann boundary condition on any 0th order flow variable translates to a Neumann condition on the 1 st order variable. A Dirichlet condition on a 0 th order flow variable does not allow a change in that quantity, so that the corresponding 1st order variable value becomes zero on that boundary. A seal-specific inlet boundary condition where a stagnation 
pressure and entrance loss factor is specified translates as:

$$
\begin{aligned}
& p_{b}=p_{0}-\frac{1}{2} \rho u^{2}(1+\xi) \\
& \widehat{p}_{1 b}=-\rho u \hat{u}_{1}(1+\xi)
\end{aligned}
$$

where $u$ is the Cartesian velocity along the axis of the seal, $p_{O}$ is the specified stagnation pressure, $\xi$ is the loss coefficient, and subscript $b$ refers to the value to be applied at the inlet boundary.

\section{Calculation of Rotordynamic Coefficients}

To calculate the rotordynamic coefficients, the fluid reaction forces on the rotor wall are needed. These are obtained by integrating the 1 st order pressure solutions on the rotor wall, and then the total force is resolved along the $\mathrm{y}$ and $\mathrm{z}$ directions to yield the complex reaction forces $F_{y 1}$ and $F_{z 1}$. Using the definition of the rotor center location, Equation 3, expressions for the rotor center velocity and accelerations are found, and then substituted in the definition of the rotordynamic coefficients, Eqn. 4. Separating out sine and cosine terms, the reaction forces are linked to rotordynamic coefficients as:

$$
\begin{aligned}
& -F_{y 1(\text { real })}=K_{y y}-C_{y z} \Omega-M_{y y} \Omega^{2} \\
& -F_{y 1(\text { imag })}=K_{y z}-C_{y y} \Omega-M_{y z} \Omega^{2} \\
& -F_{z 1 \text { (real })}=-K_{z y}+C_{z z} \Omega+M_{z y} \Omega^{2} \\
& -F_{z 1 \text { (imag) }}=K_{z z}+C_{z y} \Omega-M_{z z} \Omega^{2}
\end{aligned}
$$

To evaluate the individual coefficients, the variation of reaction forces with whirl frequency is needed. This is done by calculating the 1 st order solutions at several different whirl frequencies. Appropriate curves are then fit (2nd order polynomials) to the force Vs. frequency relations to obtain the complete set of rotordynamic coefficients.

\section{NUMERICAL RESULTS}

Two test cases are presented here to demonstrate the capability and accuracy of the perturbation method. In both cases experimental and/or published numerical data is used to validate the method.

\section{Long Incompressible Flow Annular Seal}

Experimental data for a long annular seal with incompressible flow was reported by Kanemori and Iwat-

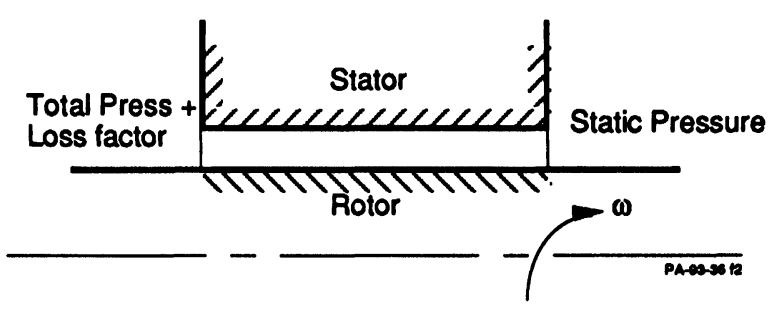

FIGURE 2 Boundary conditions for the annular seal test cases

subo[1992]. The seal dimensions were: length $=240$ $\mathrm{mm}$., rotor radius $=39.656 \mathrm{~mm}$., clearance $=0.394$ $\mathrm{mm}$., entrance loss factor $=0.5$. The stator is nominally concentric, and results in a skew-symmetric set of coefficients. The boundary conditions were (Figure 2): specified static pressure at seal exit, specified pressure and loss coefficient at inlet. Experimental measurements of inlet swirl were imposed at the inlet boundary. Solutions were obtained at four different rotor spin speeds: $600,1080,1800$ and $3000 \mathrm{rpm}$, and at seal pressure differentials $(\Delta \mathrm{P})$ of $20,50,100,250,500$ and $900 \mathrm{KPa}$. The low-Re $\mathrm{k}-\epsilon$ model was used and the computational grids used had 15 cells in the axial direction, 30 in the circumferential direction and 12 or 16 cells in the radial direction, depending on the pressure differential applied. Central differencing with 0.1 damping was used for spatial discretization of convective terms. The results of the calculations are shown in Figures 3 through 7 . The experimental data are also plotted on these plots, and very good correlation between the two sets is obtained. The discrepancy in $\mathrm{K}$ value at $1980 \mathrm{rpm}$ and $900 \mathrm{KPa}$ pressure is probably due to the

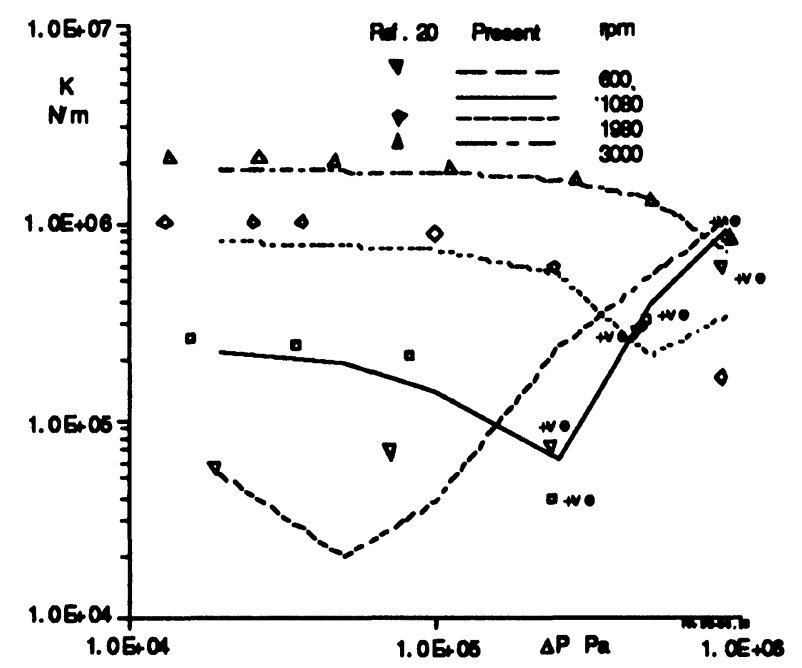

FIGURE 3 Direct stiffness coefficient, long annular seals; all unmarked values are -ve 


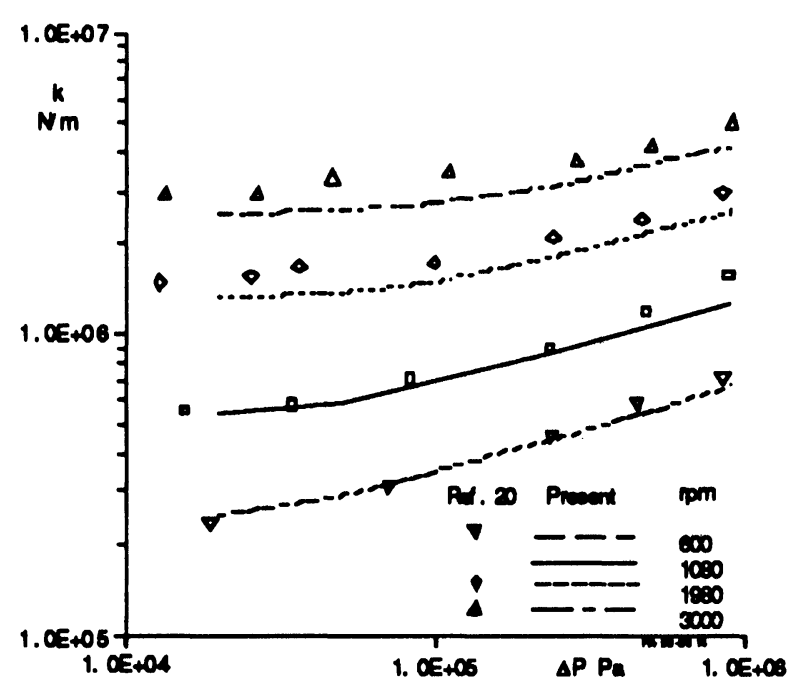

FIGURE 4 Cross coupled stiffness coefficients, long annular seal

low-Re k- $\epsilon$ turbulence model. The computed stiffness coefficients $\mathrm{K}, \mathrm{k}$, inertia coefficients $\mathrm{M}, \mathrm{m}$, and crosscoupled damping coefficients $\mathrm{C}$, $\mathrm{c}$, were found to be within $10 \%$ to $15 \%$ of the experimental values for a majority of the data points. The direct damping coefficients, however showed somewhat higher discrepancies (up to about $40 \%$ ) in the lower $\Delta \mathrm{P}$ values and at higher rpm. This long seal exhibits negative direct stiffness coefficients at high rotor rpm over the entire $\Delta \mathrm{P}$ range, as well as at low pressure differentials at the lower rpm. The numerical results predict the negative stiffness as well as the crossover to positive values at higher seal pressure differentials $\Delta \mathrm{P}$ and low rpms.

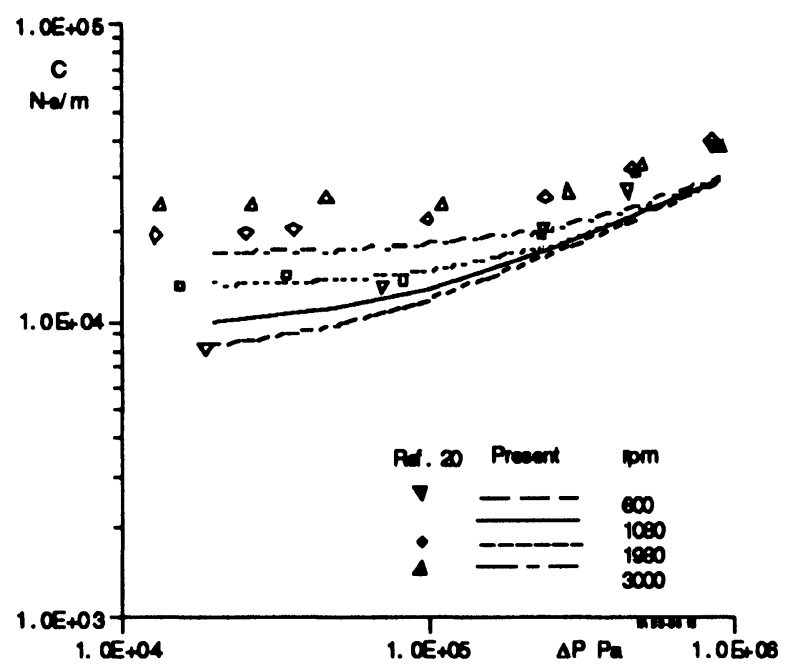

FIGURE 5 Direct damping coefficients, long annular seal

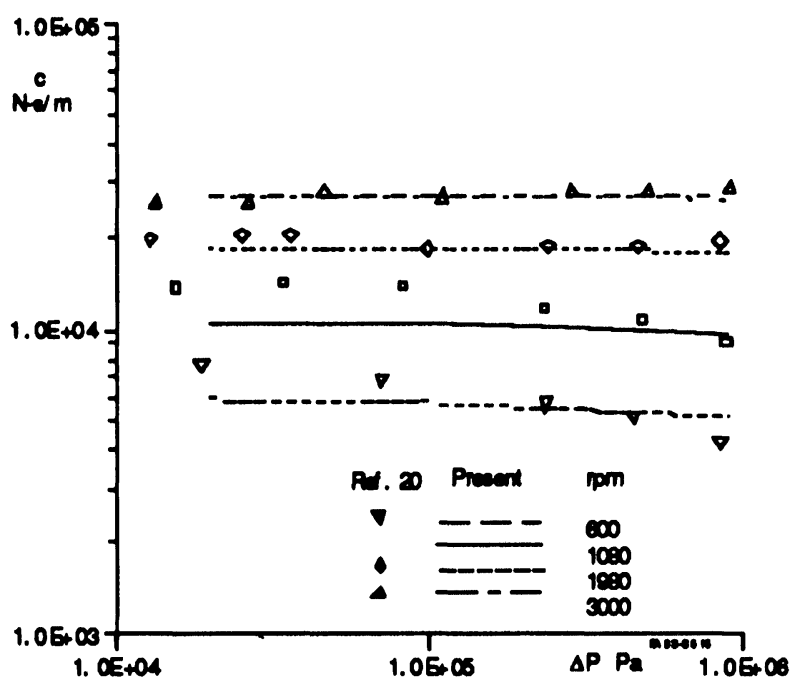

FIGURE 6 Cross coupled damping coefficients, long annular seal

The analysis given by Tam et.al. [1988] was applied to a few data points from the present computations to check the ability of the perturbation model to predict flow physics as outlined in their paper. Seal pressure differentials of 50 and $500 \mathrm{KPa}$ at a rotor speed of $1080 \mathrm{rpm}$ were selected as the two sample points. In the present set of calculations, the real parts of the reaction forces $F_{y}$ and $F_{z}$ correspond to the direct and quadrature dynamic stiffness as described in Tam et.al. [1988].

Plot of the quadrature dynamic stiffness, $\mathrm{K}_{\mathrm{t}}$ Vs. the whirl frequency $\Omega$ for the two pressure differentials is shown in Figure 8 . The curves are straight lines that indicate that the rotordynamic coefficients are indepen-

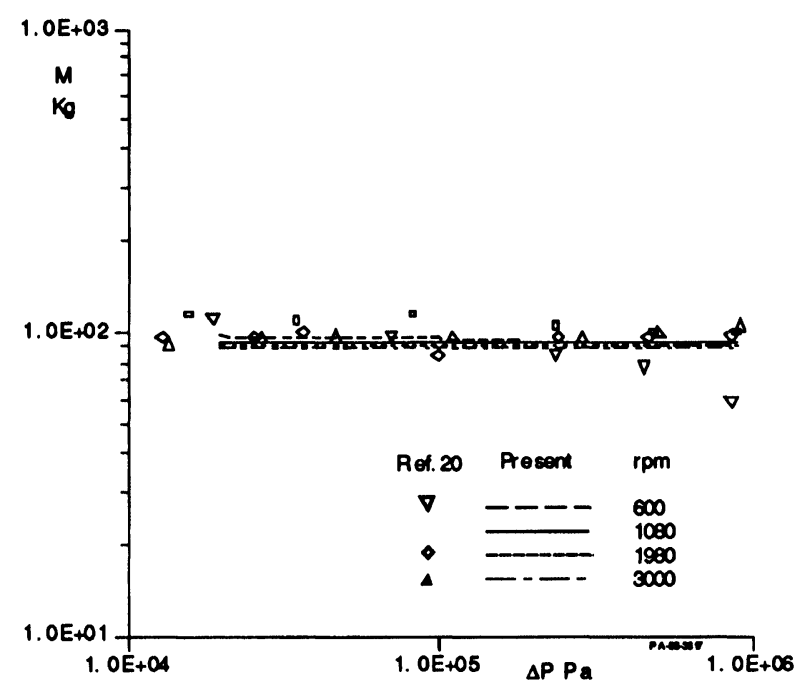

FIGURE 7 Direct inertia (mass) coefficients, long annular seal 


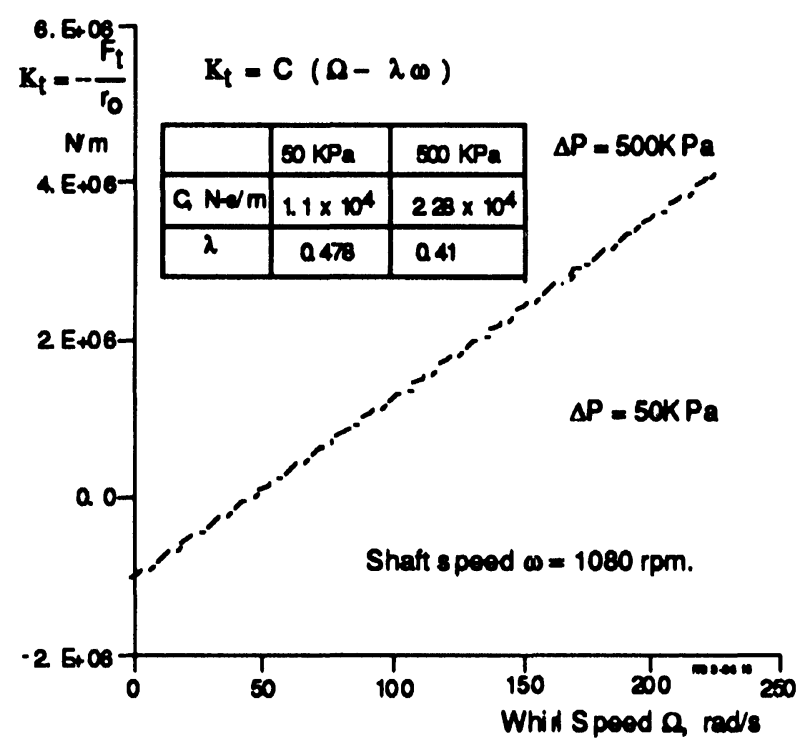

FIGURE 8 Quadrature Dynamic Stiffness Vs. Whirl frequency

dent of the whirl frequency in these runs. Values of the average circumferential velocity ratio, $\lambda$, for the 50 and $500 \mathrm{KPa}$ cases are 0.478 and 0.412 respectively. This corresponds to a spin-up of the fluid in the seal from specified inlet $\lambda$ values of 0.46 and 0.32 (from experimental data). This inlet swirl generates positive values for the so called cross-coupled stiffness coefficient $\mathrm{k}$.

The direct dynamic stiffness is a function of the modified whirl speed $(\Omega-\lambda \omega)$, and Figure 9 shows the plots. Slopes of the lines give the direct inertia coefficient M. In the model described by Tam et.al. [1988], the direct stiffness coefficient is defined as the intercept on the vertical axis in Fig. 9; and is positive for both $\Delta \mathrm{P}$ values. However, as defined in Eqn. 2 in the present calculations, the direct stiffness coefficient $\mathrm{K}$ is related to the intercept $K_{r, O}$ as

$$
K=K_{r, 0}-M \lambda^{2} \omega^{2} .
$$

The negative $\mathrm{K}$ for $\Delta \mathrm{P}=50 \mathrm{KPa}$ in the present calculations results due to the inertia term, and a small intercept $K_{r, 0}$, while the larger intercept at $500 \mathrm{KPa}$ results in a net positive $K$.

\section{Annular Eccentric Seal}

This case involves an annular seal with incompressible flow, with nominal static eccentricity ratios varying from 0 to 0.7 . The seal dimensions were: seal length $=40$ mm., seal radius $=80 \mathrm{~mm}$., clearance $=0.36 \mathrm{~mm}$., entrance loss factor $=0.5$. The boundary conditions were similar to those before. Experimental data for the stiffness coefficients was reported by Falco et. al [1986], and a compilation of various numerical data by Simon and Frene [1991]. Solutions were obtained at a rotor speed of $4000 \mathrm{rpm}$, average preswirl of 0.3 , a pressure differential of $1.0 \mathrm{Mpa}$, and at several static eccentricity ratios from 0.0 to 0.7 . The standard $k-\epsilon$ model with wall functions was used and the grid used had 12 cells in the axial, 6 in radial and 30 in the circumferential direction. Central differencing with 0.1 damping was used for convective terms. Results of these calculations are shown in Figures 10 through 19. The experimental as well asnumerical data is also plotted for comparison, and very good

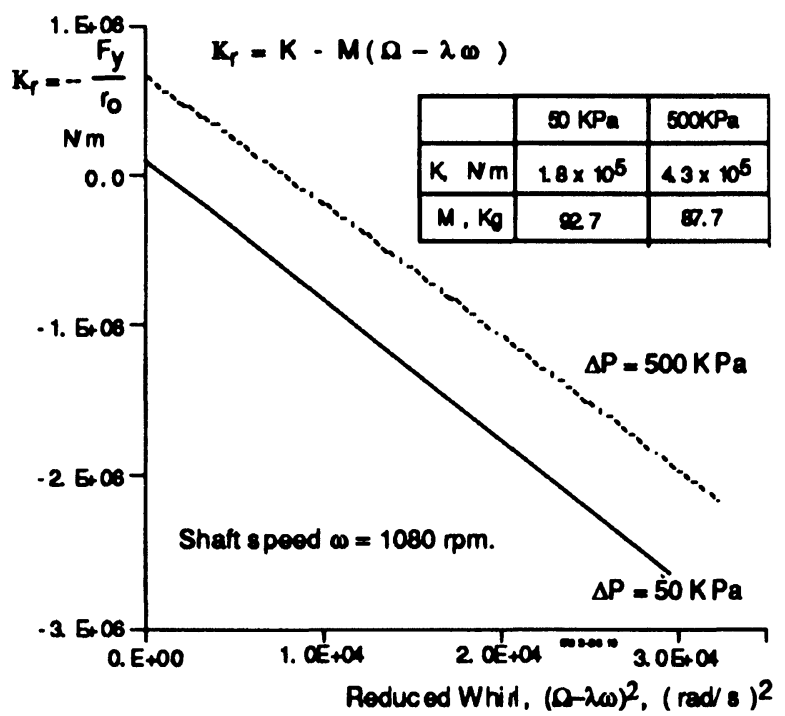

FIGURE 9 Direct Dynamic Stiffness Vs. Reduced Whirl Frequency

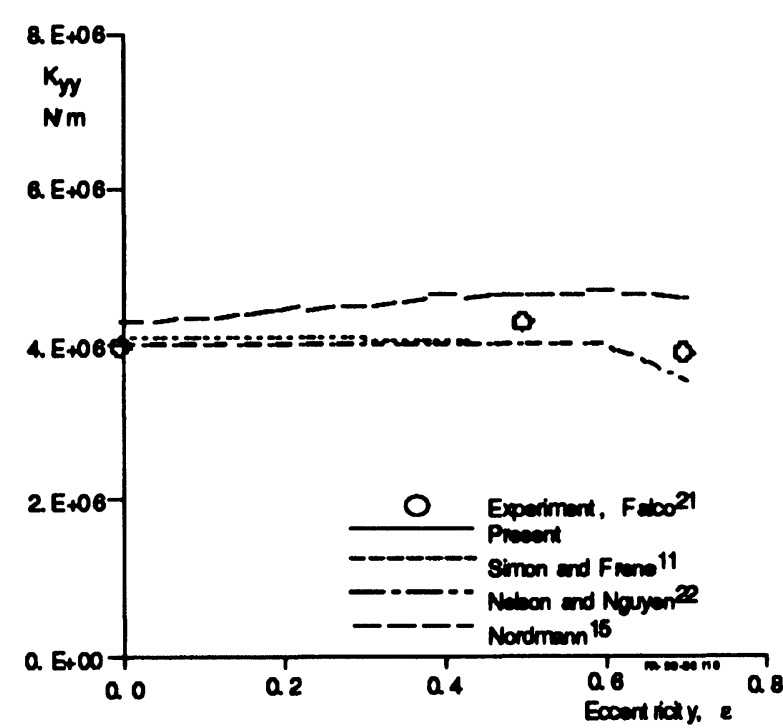

FIGURE 10 Direct stiffness coefficient, $\mathrm{K}_{\mathrm{yy}}$ eccentric annular seal 


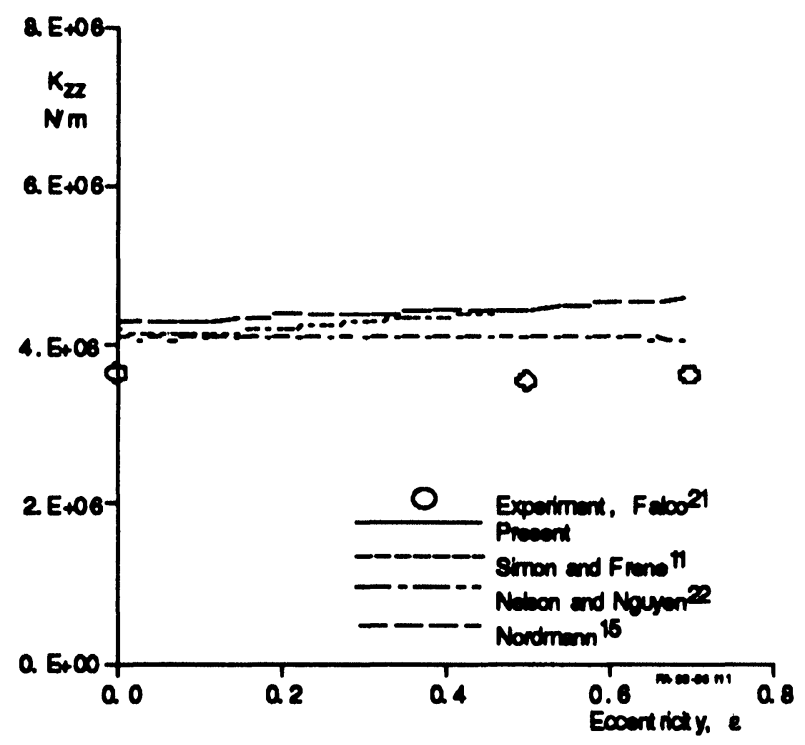

FIGURE 11 Direct stiffness coefficient, $\mathrm{K}_{\mathrm{zz}}$, eccentric annular seal

correlation between the present results and published experimental and numerical data is seen.

\section{CONCLUDING REMARKS}

A small-perturbation method for rotordynamic coefficients of seals has been developed. The method is based on the perturbations in the 3-D, Navier Stokes equations for BFC grids. The method has the capability of treating

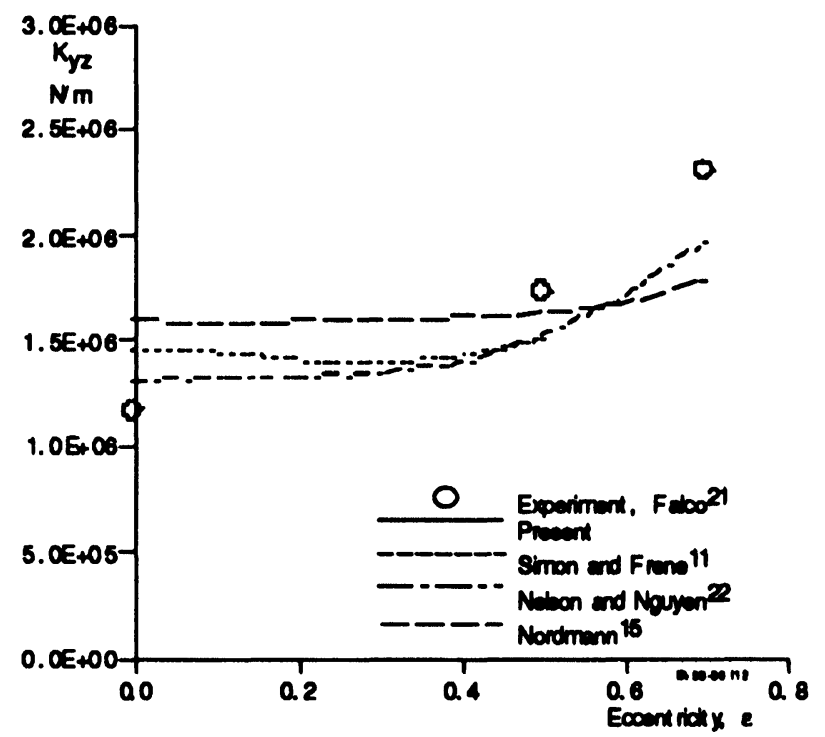

FIGURE 12 Cross coupled stiffness coefficient, $\mathrm{K}_{\mathrm{yz}}$, eccentric annular seal

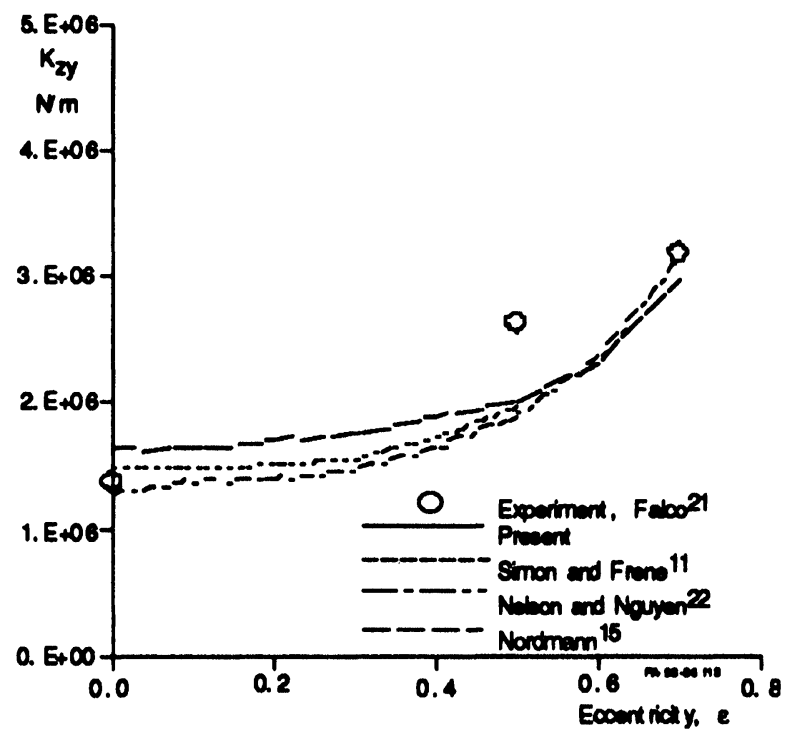

FIGURE 13 Cross coupled stiffness coefficient, $\mathrm{K}_{\mathrm{zy}}$, eccentric annular seal

nominally eccentric and/or misaligned rotors. Solutions presented here included incompressible flow concentric and eccentric annular seals. In the concentric seal, numerical results correlate well with experimental data, including the negative direct stiffness that result in this long annular seal. For the eccentric seal case, the present results were compared with experimental and other numerical data, and again good comparison between thetwo was obtained for all coefficients except one cross-coupled damping coefficient.

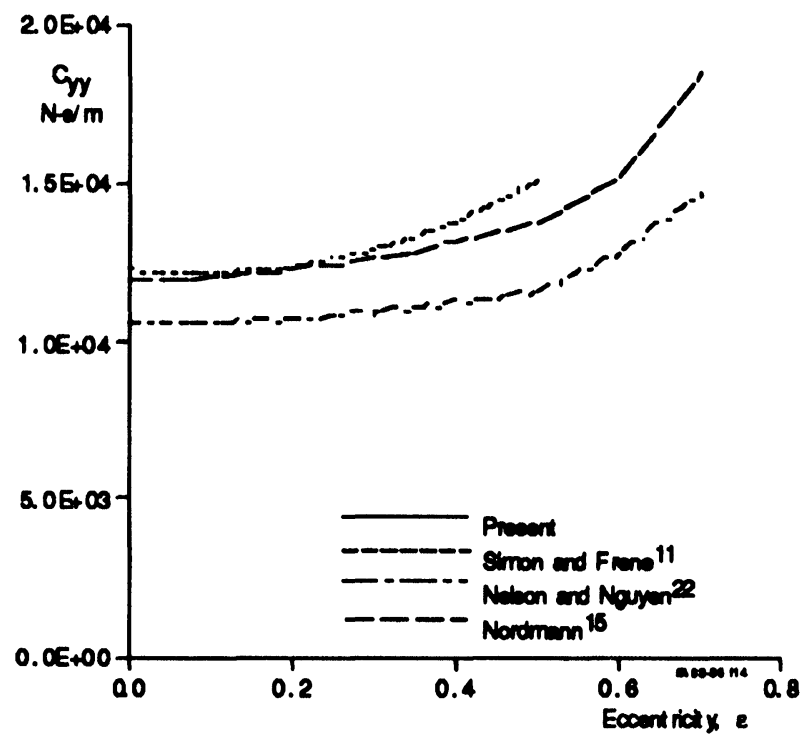

FIGURE 14 Direct damping coefficient, $\mathrm{C}_{\mathrm{yy}}$, eccentric annular seal 


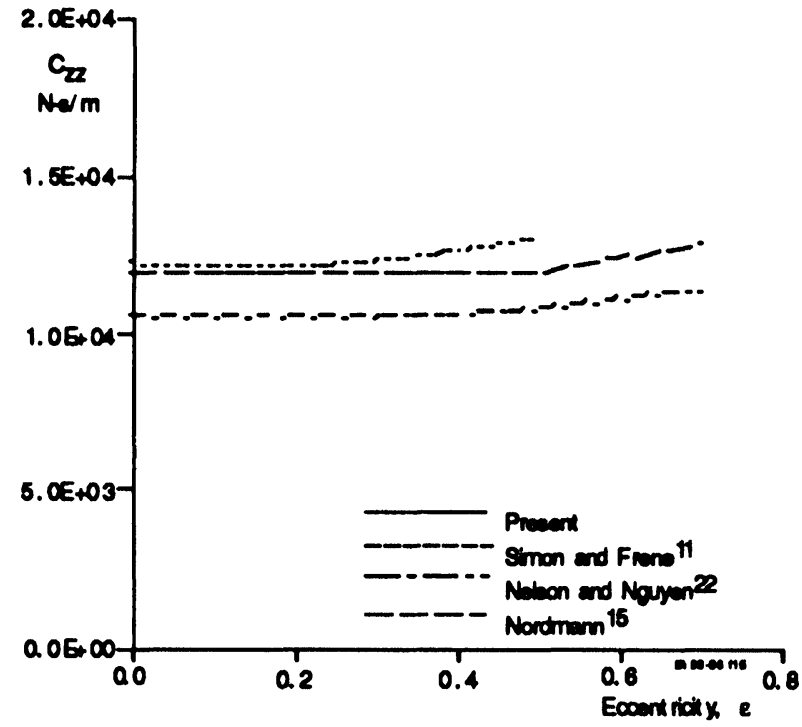

FIGURE 15 Direct damping coefficient, $\mathrm{C}_{\mathrm{zz}}$, eccentric annular seal

The use of 3-D BFC grids and $\mathrm{N}-\mathrm{S}$ equations allow this model to treat a wide range of seals, including seals such as labyrinth, grooved, tip and stepped. This method can also be easily extended to compute interactions between fluid flow and larger rotating components, e.g. shrouded impellers. Current plans include extension to compressible flows and more complicated geometry seals.

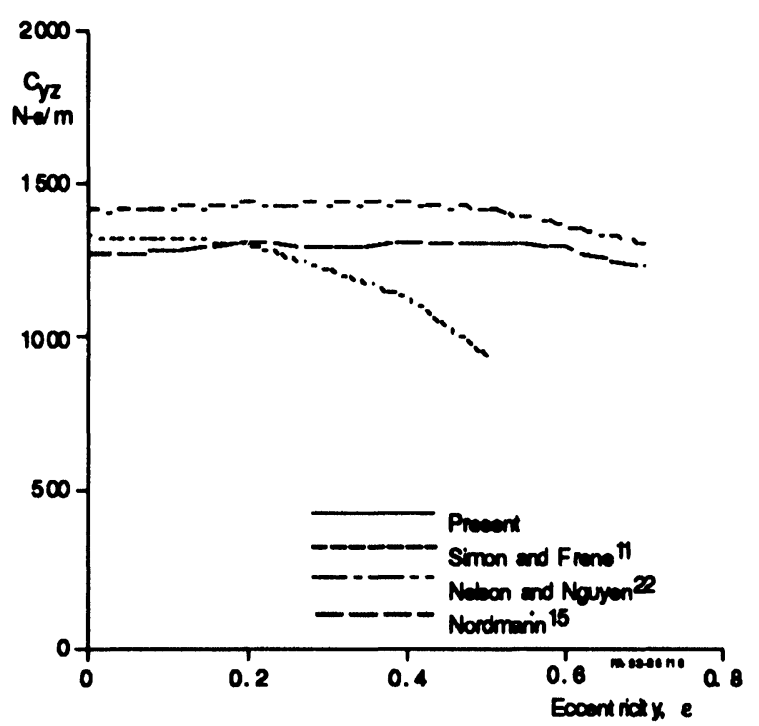

FIGURE 16 Cross coupled damping coefficient, $\mathrm{C}_{\mathrm{yz}}$, eccentric annular seal

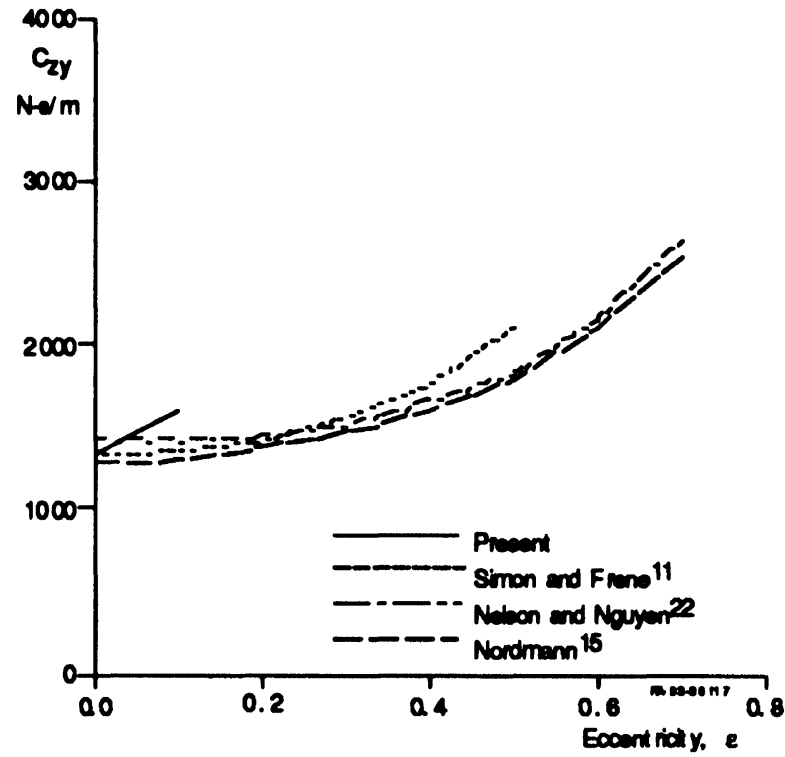

FIGURE 17 Cross coupled damping coefficient, $\mathrm{C}_{\mathrm{zy}}$, eccentric annular seal

\section{Acknowledgements}

This work was performed under NASA LeRC contract No. NAS3-25644, entitled Study of Fluid Dynamic Forces in Seals, with Mr. R.C. Hendricks as the Technical Monitor. This support is greatly appreciated. The authors would also like to thank Dr. Andrzej Przekwas of CFDRC for his guidance during this project.

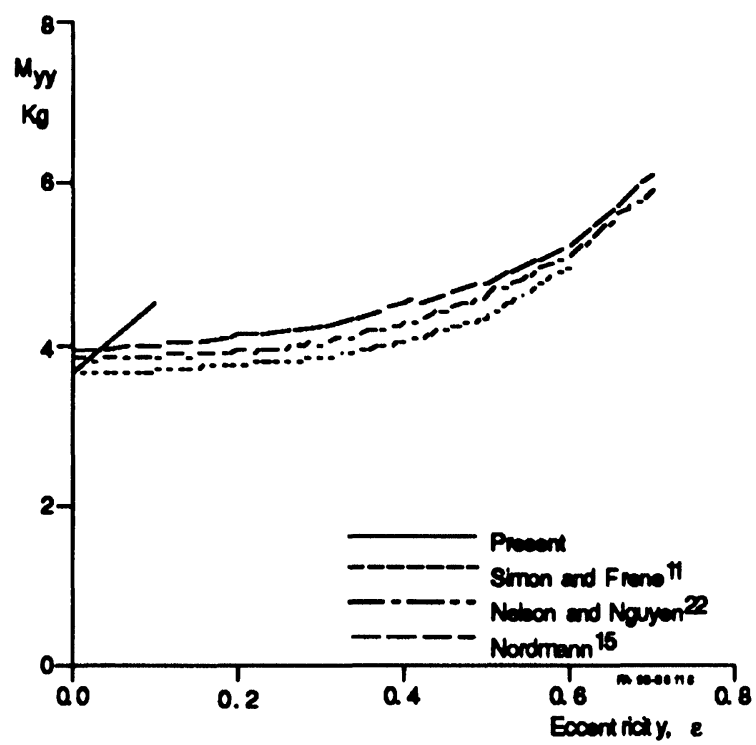

FIGURE 18 Direct inertia (mass) coefficient, $\mathrm{M}_{\mathrm{yy}}$, eccentric annular seal 


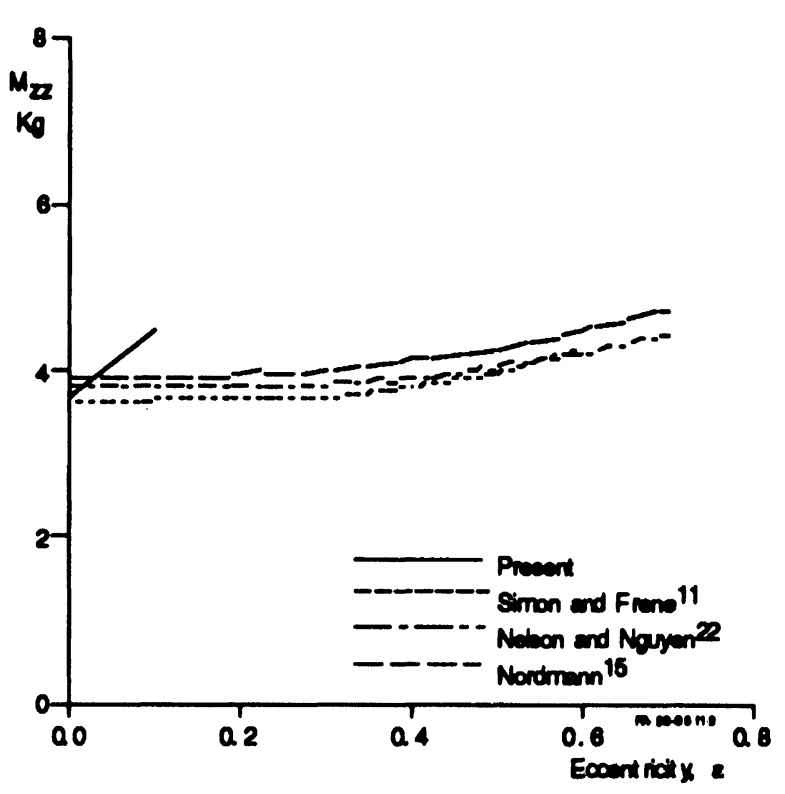

FIGURE 19 Direct inertia (mass) coefficient, $\mathrm{M}_{\mathrm{zz}}$, eccentric annular seal

$\begin{array}{ll}\text { Nomenclature } & \\ C_{y y}, C_{y z}, C & \text { direct damping coefficients, N-s/m } \\ C_{y z}, C_{z y}, C & \text { cross coupled damping coefficients, N-s/m } \\ e & \text { small number, perturbation parameter } \\ F & \text { Force, } \mathrm{N} \\ g^{j k} & \text { elements of metric tensor } \\ i & \text { complex axis }-1 \\ J & \text { Jacobian of transformation } \\ K_{y y}, K_{z z}, K & \text { direct stiffness coefficients, } \mathrm{N} / \mathrm{m} \\ K_{y z}, K_{z y}, k & \text { cross-coupled stiffness coefficients, } \mathrm{N} / \mathrm{m} \\ M_{y y}, M_{z z}, M & \text { direct inertia (mass) coefficients, Kg } \\ M_{y z}, M_{z y}, m & \text { cross-coupled inertia coefficients, } \mathrm{Kg} \\ p & \text { pressure, N/m }{ }^{2} \\ r_{o} & \text { length scale for whirl radius, } \mathrm{m} \\ S & \text { source term in momentum equation, } \mathrm{N} \\ t & \text { time, } \mathrm{s} \\ u, v, w & \text { Cartesian velocities in } \mathrm{x}, \mathrm{y} \text { and } \mathrm{z} \text { directions, } \\ & \text { fh/id velocity vector, } \mathrm{m} / \mathrm{s} \\ g & \text { complex grid velocity vector, m/s } \\ y, z & \text { rotor center displacement, } \mathrm{m} \\ y_{o}, z_{o} & \text { nominal coordinates for rotor center, } \mathrm{m} \\ \dot{y}, \dot{z} & \text { rotor center velocity, } \mathrm{m} / \mathrm{s} \\ \ddot{y}, \ddot{z} & \text { rotor center acceleration, } \mathrm{m}^{2} / \mathrm{s}\end{array}$

$\begin{array}{ll}\text { Greek } & \\ \epsilon & \text { turbulence dissipation } \\ \epsilon^{k} & \text { contravariant base vectors, } \mathrm{m} \\ \lambda & \text { circumferential velocity ratio } \\ \xi & \text { entrance loss factor } \\ \xi, \eta, \zeta, \xi^{k} & \text { coordinate direction in body fitted grids, } \mathrm{m} \\ \rho & \text { density, } \mathrm{kg} / \mathrm{m}^{3} \\ \tau & \text { transformed time variable, } \mathrm{s}\end{array}$

Subscripts
0
1
$1 c$
$1 s$
$g$
$k$
$x, y, z$
(imag)
(real)

\section{Superscripts}

$\wedge$

1

$i, j, k$
Cartesian velocity representation in momentum equation, $\mathrm{m} / \mathrm{s}$ whirl frequency, $\mathrm{rad} / \mathrm{s}$

\author{
zeroeth order quantities \\ first order quantities \\ first order quantity associated with $(\cos \Omega t)$ \\ first order quantity associated with $(\sin \Omega \mathrm{t})$ \\ grid \\ coordinate index \\ force component in Cartesian directions \\ imaginary part of a complex quantity \\ real part of a complex quantity
}

complex 1st order variable contravariant direction

vector

index for coordinate directions

\section{References}

Alford, J.S., October 1965. Protecting Turbomachinery from selfexcited rotor whirl, Trans. ASME, J. of Engineering for Power, pp. 333-344. Instability Problems in High Performance Turbomachinery, Texas.

Athavale, M.M., Przekwas, A.J., and Hendricks, R.C., 1992. A Finite Volume Numerical Method to Calculate Fluid Forces and Rotordynamic Coefficients in Seals, AIAA Paper 92-3712, Presented at the AIAA Joint Propulsion conference, Nashville.

Baskharone, E.A., and Hensel, S.J., 1991. A Finite-Element Perturbation Approach to Fluid/Rotor Interaction in Turbomachinery Elements. Part 1: Theory, Trans. ASME, J. of Fluids Engineering, V. 113, pp. 353-361.

Baskharone, E.A., and Hensel, S.J., 1991. A Finite-Element Perturbation Approach to Fluid/Rotor Interaction in Turbomachinery Elements. Part 2: Application, Trans. Trans. ASME, J. of Fluids Engineering, V. 113, pp. 362-367.

Black, H.F., 1969. Effects of Hydraulic Forces in Annular Pressure Seals on the Vibrations of Centrifugal Pump Rotors, J. of Mechanical Engineering Science V. II, No. 2.

Black H.F., and Jensen, D.N., 1970. Dynamic Hybrid Bearing Characteristics of Annular Controlled Leakage Seals, Proceedings of the Institution of Mechanical Engineers, V. 184.

Childs, D.W., 1983. Dynamic Analysis of Turbulent Annular Seals Based on Hirs' Lubrication Equation, Trans. ASME, J. of Lubrication Technology, V. 105, pp. 429-436.

Childs, D.W., 1983. Finite-Length Solutions for Rotordynamic Coefficients of Turbulent Annular Seals, Trans. ASME, J. of Lubrication Technology, V. 105, pp. 437-445.

Childs, D.W., Nelson, C.E., Nicks, C., Scharrer, J., Elrod, D., and Hale, K., 1986. Theory Versus Experiment for the Rotordynamic Coefficients of Annular Gas Seals: Part 1-Test Facility and Apparatus, Trans. ASME, J. of Tribology, V. 108, pp. 426-432.

Dietzen, F.J., and Nordmann, R., 1987. Calculating Rotordynamic Coefficients of Seals by Finite-Difference Techniques, Trans. ASME, J. of Tribology, V. 109, pp. 388-394.

Falco, M, Mimmi, G., and Mareno, G., 1986. Effects of Seals on 
Rotordynamics, Proceedings of the International Conference on Rotordynamics, Japan.

Hirs, G.G., April 1973. A Bulk-Flow Theory for Turbulence in Lubricant Films, Trans. ASME, J. of Lubrication Technology, pp. 137-146.

Kanemori, Y., and Iwatsubo, T., 1992. Experimental Study of Dynamic Fluid Forces and Moments for a Long Annular Seal, Trans. ASME, $J$. of Tribology, Vol. 114, pp. 773-778.

Nelson, C.C., 1985. Rotordynamic Coefficients for Compressible Flow in Tapered Annular Seals, Trans. ASME, J. of Tribology, V107, pp. 318-325.

Nordmann, R., and Dietzen, F.J., 1988. Finite Difference Analysis of Rotordynamic Seals Coefficients for an Eccentric Shaft Position, NASA CP-3026, Rotordynamic Instability Problems in High Performance Turbomachinery, Texas.

Nordmann, R., Dietzen, F.J., and Weiser, H.P., 1989. Calculation of Rotordynamic Coefficients and Leakage for Annular Gas Seals by Means of Finite Difference Techniques, Trans. ASME, J. of Tribology, V. 109 , pp. 545-552.
San Andres, L.A., 1991. Analysis of Variable Fluid Properties, Turbulent Annular Seals, Trans. ASME, J. of Tribology, Vol. 113, pp. 694-702.

Simon, F. and Frene, J., 1989. Static and Dynamic Characteristics of Turbulent Annular Eccentric Seals: Effect of Convergent-Tapered Geometry and Variable Fluid Properties, Trans. ASME, J. of Tribology, V. 111, pp. 378-385.

Simon, F., and Frene, J., 1991. Analysis for Incompressible Flow in Annular Pressure Seals, ASME Paper 91-Trib-50, STLE/ASME Tribology Conference.

Tam, L.T., Przekwas, A.J., Muszynska, A., Hendricks, R.C., Braun, M.J., and Mullen, R.L., 1988. Numerical and Analytical Study of Fluid Dynamic Forces in Seals and Bearings, Trans. ASME, J. of Vibration, Acoustics, Stress and Reliability in Design, V. 110, pp. 315-325.

Von Pragenau, G.L., 1987, 1982. Damping Seals for Turbomachinery, NASA Technical Paper. 

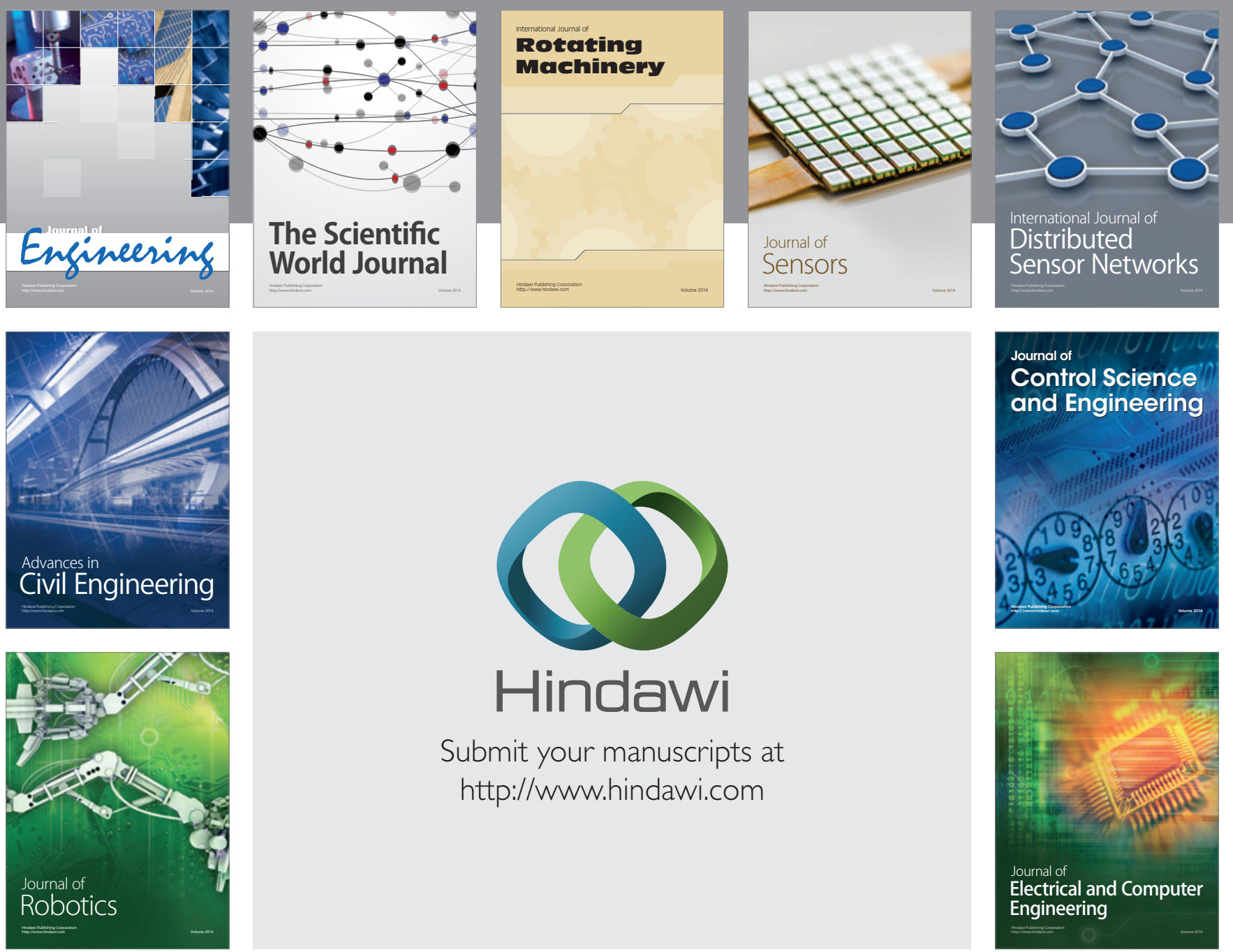

Submit your manuscripts at

http://www.hindawi.com
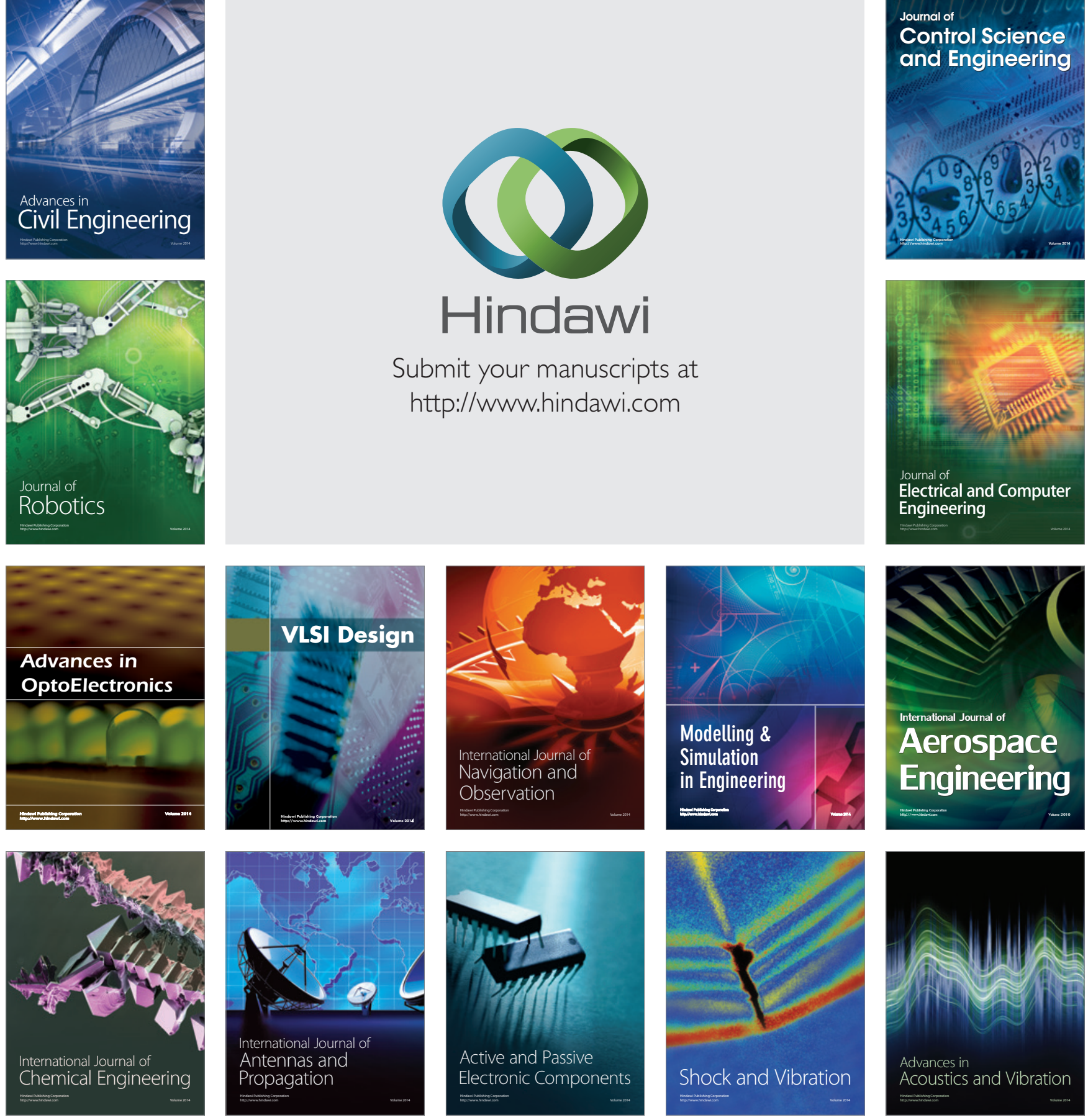